\title{
Minimal Information for Neural Electromagnetic Ontologies (MINEMO): A standards-compliant method for analysis and integration of event-related potentials (ERP) data
}

\author{
Gwen Frishkoff', 2, 3 Jason Sydes ${ }^{4}$, Kurt Mueller ${ }^{4}$, Robert Frank², Tim Curran ${ }^{3,5}$, \\ John Connolly ${ }^{3,6}$, Kerry Kilborn ${ }^{3,7}$, Dennis Molfese ${ }^{3,8}$, Charles Perfetti ${ }^{3,9}$, \& Allen Malony ${ }^{2,4}$ \\ ${ }^{1}$ Department of Psychology \& Neuroscience Institute, Georgia State University, Atlanta, GA \\ ${ }^{2}$ Neurolnformatics Center, University of Oregon, Eugene, OR \\ ${ }^{3}$ Neural ElectroMagnetic Ontologies (NEMO) ERP Consortium \\ ${ }^{4}$ Department of Computer \& Information Science, University of Oregon, Eugene, OR \\ ${ }^{5}$ Department of Psychology \& Neuroscience, University of Colorado, Boulder, CO \\ ${ }^{6}$ Department of Psychology and Linguistics, McMaster University, Ontario, CA \\ ${ }^{7}$ Department of Psychology, Glasgow University, Scotland, UK \\ ${ }^{8}$ Department of Psychology, University of Nebraska-Lincoln, Lincoln, NE \\ ${ }^{9}$ Department of Psychology and Linguistics, University of Pittsburgh, Pittsburgh, PA
}

\begin{abstract}
We present MINEMO (Minimal Information for Neural ElectroMagnetic Ontologies), a checklist for the description of event-related potentials (ERP) studies. MINEMO extends MINI (Minimal Information for Neuroscience Investigations)to the ERP domain. Checklist terms are explicated in NEMO, a formal ontology that is designed to support ERP data sharing and integration. MINEMO is also linked to an ERP database and web application (the NEMO portal). Users upload their data and enter MINEMO information through the portal. The database then stores these entries in RDF (Resource Description Framework), along with summary metrics, i.e., spatial and temporal metadata. Together these spatial, temporal, and functional metadata provide a complete description of ERP data and the context in which these data were acquired. The RDF files then serve as inputs to ontology-based labeling and meta-analysis. Our ultimate goal is to represent ERPs using a rich semantic structure, so results can be queried at multiple levels, to stimulate novel hypotheses and to promote a high-level, integrative account of ERP results across diverse study methods and paradigms.
\end{abstract}

Keywords: Ontology, database, data sharing, standardization, experiment metadata, neuroscience, electrophysiology, event-related potentials

\section{Introduction}

Over the last few decades, neuroscience has witnessed an explosion of methods for the measurement of human brain function, including high-density (multi-sensor) event-related potentials (ERPs). In comparison with other techniques, the ERP method has several advantages: it is completely safe and noninvasive, it is inexpensive and portable, and - unlike methods such as functional magnetic resonance imaging (fMRI) - it is a direct measure of neuronal activity. The ERP method also has excellent (millisecond) temporal resolution, which is critical for representation of neural dynamics. Remarkably, despite these many virtues, there are few quantitative comparisons ("meta-analyses") of ERP results, reflecting the complexity of ERP data and the wide variety of methods that are used to extract and analyze ERP metadata [1-3].

To address this gap, we have gathered an interdisciplinary team of researchers in informatics and human neuroscience to form project NEMO (Neural ElectroMagnetic Ontologies). Our neuroscience experts are internationally known for their ERP studies of language and cognition and have partnered to form a consortium. Consortium members provide ERP datasets and contribute to the design and testing of ERP ontologies and ontology-based methods for meta-analysis [3]. 
In the present paper, we present a minimal information checklist, called MINEMO (Minimal Information for NEMO). MINEMO specifies the key information that should be provided when an ERP experiment is uploaded to the NEMO database. MINEMO terms are explicated in the NEMO ontology, a formal semantic system that we have created for the ERP domain. We have also developed a web application (the NEMO portal) and database, which are aligned with the MINEMO checklist and ontology. Together, the checklist, ontology, and database are intended to support the first complete, cross-laboratory meta-analysis for the ERP domain.

The rest of this paper is structured as follows. In Section 2, we outline prior work on the development of minimal information (MI) checklists, controlled vocabularies, and formal semantic systems (ontologies). In Section 3, we present the MINEMO checklist. In Section 4 we describe how MINEMO is aligned with the NEMO ontology and how it is linked to the NEMO database and portal. Section 5 provides a brief a summary and describes ongoing and future work.

\section{Related work}

In this section we describe prior work that has informed the development of MINEMO. This work falls into three categories: Standardized checklists, which specify key ("minimal") information for representation of data in a particular domain; (2) Controlled vocabularies, which prescribe standard terms, together with human-readable definitions, for consistent annotation of data; and (3) Formal ontologies, which include defined classes, class hierarchies, relations between classes, and axioms for reasoning over class- and instance-level information.

\section{Standardized Checklists}

The Minimum Information for Biological and Biomedical Investigations (MIBBI) is a pioneering project that aims to coordinate guidelines for reporting of scientific metadata across domains [4]. Central to this effort is the MIBBI portal, a clearinghouse for proposed MI checklists. The motivation for MIBBI is two-fold: (1) to promote the use of standard checklists by various stakeholders (e.g., journals, authors, reviewers, and funders), and (2) to facilitate "harmonization," that is, mapping or integration, of domain-specific guidelines. To the extent that researchers can agree on these guidelines, the MIBBI effort may constitute an important first step towards widespread data sharing within and across biological domains.

One checklist that is available through the MIBBI portal is the Minimal Information for Neuroscience Investigations, or MINI, checklist [5]. MINI specifies guidelines for reporting of electrophysiology experiments and comprises eight sets of fields (i.e., tables): (1) General features of an experiment, (2) Study subject(s), (3) Anatomical location of electrophysiological recording, (4) Experimental task, Experimental stimuli, (6) Behavioral response data, (7) Recording specifications and (8) Electrical (time series) data. MINI is intended to cover a wide range of electrophysiological protocols, but appears best suited for reporting on single-cell recordings, as opposed to far-field recordings, such as EEG and ERPs.

In human neuroscience, Poldrack and associates have proposed a set of standards for reporting of fMRI data, called MIfMRI (see MIBBI portal and Appendix A in Ref [6]). MIfMRI specifies minimal information about human subjects, a useful complement to MINI, and categories such as Task and Behavioral performance, which are available in MINI and can be readily extended to other types of human neuroscience protocols (e.g., ERP experiments). Other categories, such as experimental design, appear more narrowly suited for description for fMRI experiments.

There are several publications on ERP research design, implementation, and reporting of results [7-9], but no minimal information checklists or similar resources for the ERP domain. In 2000, Picton and associates provided a detailed and highly influential set of guidelines [9]. In developing MINEMO, we have taken these guidelines under consideration. At the same time, we have tried to create a usable (i.e., relatively short) checklist, comprising no more than $\sim 60$ fields - and no more than $\sim 20$ that must be completed before data are uploaded to the NEMO database. In this respect, we follow BrainMap and MIBBI researchers, who have discussed lessons learned in developing metadata tools and resources and then working to secure buy-in from users $[4,10]$. However good the resource, it is unlikely to find widespread use if it is clunky or time-consuming to use. 


\section{Controlled Vocabularies}

For the NEMO project, we need consistent annotation of ERPdata, since we are aiming to conduct cross-lab meta-analysis. MI checklists can promote the use of consistent guidelines for reporting of studydata. However, there is no guarantee that different researchers will use the same terms for data mark-up. For this reason, researchers in several domains have created controlled vocabularies, or lexicons, for data annotation $[11]^{1}$.

In human neuroscience, the BrainMap lexicon has enjoyed widespread use, particularly in connection with their database $[10,12]$. The BrainMap database is an immense repository, resulting from more than 10 years of work curating results from thousands of functional brain imaging studies. Making such a collection reliably searchable requires consistent and precise naming of study information. To this end, the BrainMap team has created a portal called 'Sleuth' that supports controlled entry of metadata. The BrainMap lexicon (aka the 'MetaData Coding Scheme') covers a range of metadata, including stimuli, tasks (instructions), and protocols for measurement of behavioral and brain responses. In addition to historical (and often idiosyncratic) terms for paradigms, such as the 'Stroop Task' or 'Auditory Oddball Task', each set of results that is entered in BrainMap is linked to a specific Stimulus, Task (Instructions), and Response category. Recent studies have used data mining to uncover patterns of brain activation across different paradigms that share stimulus, task, and/or response properties, demonstrating the utility of fine-grained, consistent annotation of experiments [13].

\section{Formal Ontologies}

A recent trend in bio- and neuro-informatics is the creation of domain ontologies [14]. Like a controlled vocabulary, an ontology contains semantic categories or classes that refer to welldefined entities (e.g., 'stimulus', 'response'). Each class has a uniform resource identifier, or URI, which is globally unique (e.g., http://purl. bioontology.org/NEMO/ontology/NEMO.owl\#NEM 0_4762000), in addition to a human-readable label (e.g., 'onset_stimulus_presentation'). In addition, ontologies specify the semantic relations between classes (e.g., 'onset_stimulus_presentation proper_part_of some presentation_of_stimulus').
These relations are called object properties and impart much of the power behind ontologies. For example, in NEMO the object property rostral_to is transitive and has an inverse property, caudal_to. Thus, the assertions '(Electrode) $\mathrm{Fz}$ rostral_to (electrode) $\mathrm{Cz}^{\prime}$ and '(Electrode) $\mathrm{Pz}$ caudal_to (electrode) Cz' support the inference that '(Electrode) $\mathrm{Fz}$ rostral_to (electrode) Pz'. Assertions can be built into the ontology (e.g., as class restrictions). When they are defined as equivalent class statements, they can serve as rules to support classification of instance-level information (e.g., scientific data).

In NEMO, ERP patterns are associated with rules that specify the spatial, temporal, and functional (experimental) properties that are required for an ERP observation to be classified as a particular kind of pattern. In this way, the ontology becomes more than a static resource: it functions as a dynamic tool for interpretation of data in the context of a larger base of knowledge.

NEMO has adopted many of the recommended practices outlined by the OBO Foundry [15], including re-use of existing resources (checklists, ontologies, etc.), modularity or orthogonality, human-readable annotations, and - perhaps most important - use of the Basic Formal Ontology (BFO) as an upper ontology and the Ontology of Biological Investigations (OBI) as a mid-level ontology [15]. In doing so, we have joined a community of researchers who have adopted similar practices in order to facilitate collaborative development and harmonization of neuroscience resources. For example, the Neuroscience Information Framework (NIF) [1517] is a leading project that aggregates online sources of neuroscience data, including databases, web sites, publications, and XML files, and provides a search interface across these disparate sources. An essential part of this effort is the NIF ontology (NIFSTD [15]; ), which extends the older BirnLex ontology to cover additional domains, such as neurons, genetics, proteomics, and phenotypes. The BirnLex ontology has also given rise to the cognitive paradigms ontology, or cogPO [18]. CogPO is also based on BFO and OBI, and is building a formal ontology that uses the BrainMap Metadata Coding Scheme as a starting point. NEMO has been working closely with cogPO and NIF to coordinate ontology development efforts, particularly in the specification of experiment metadata. 


\section{Minimal information for NEMO (MINEMO)}

The MINEMO checklist was intended to augment other NEMO resources that are used to support cross-lab analysis, storage, and integration of ERP data. MINEMO extends MINI [5] to the ERP domain. In doing so, it re-uses (in whole or in part) all but one of the MINI tables ("recording location" is specific to invasive recordings and was replaced by information about EEG sensor layouts). We also made the following changes. First, we split the first table in MINI (General features) into three sets of metadata: Research Lab (PI, PI institution and contact information), Experiment (General Features), and Publication. The remaining tables were amended to reflect the use of human subjects, as well as key recording and analysis methods that are specific to ERP

\section{Subset of MINEMO terms that are required to save data to the NEMO portal (in addition to unique ID for each table)}

1. Research lab (General Features)
a. Institution
b. Principal investigator (PI)

2. Experiment (General features)

a. Experiment paradigm(s)

3. Publication
a. Publication type
b. DOI or File location (Path)

4. Study subjects (Group characteristics)
a. Diagnostic classification
b. Genus
c. Species
d. Age (average)
e. Gender (\#male, female subjects)
f. Handedness (\#RH, LH subjects)
g. Native language (modal)

5. Experiment condition
a. Experiment condition
b. Experiment task (Instructions)

6. Stimulus presentation

a. Target stimulus type

b. Target stimulus modality

7. Behavioral data collection
a. Response type
b. Response modality

research. The resulting checklist comprises $\sim 70$ fields (see Appendix A), enough information - we believe - to obtain a thorough, yet compact summary of ERP datasets. Each checklist item is linked to a key term, which is fully explicated that is, defined and annotated - within the NEMO ontology. Appendix B provides the NEMO URI for each of the MINEMO key terms.

NEMO consortium members have been very willing to provide the complete set of metadata for each of their datasets. In practice, though, some metadata is harder to locate, particularly for legacy datasets. We therefore decided to specify a subset of MINEMO terms that would be required for the first stage of meta-data entry through the NEMO portal (see Section 4). This subset of MINEMO terms is listed below.
8. EEG Data collection
a. Electrode array (Layout)
b. Sampling rate

9. EEG/ERP Data preprocessing
a. ERP event
b. ERP epoch length (in ms)
c. ERP baseline (pre-target)
duration
d. Offline reference

10. EEG/ERP Data file
a. Data file contents (EEG data type)
b. Data file format
c. Data file location (URI) 


\section{MINEMO tools and application}

In this section we describe how MINEMO supports our main goal for the NEMO project: to develop methods for cross-lab integration of ERP data. To achieve this goal, it was necessary to annotate data (spatial and temporal metrics) and metadata (data provenance) from ERP experiments using consistent terms.

\section{The NEMO ontology: Annotation of ERP spatial and temporal metrics}

ERP data are characteristically described in terms of intensity (in microvolts), distributed over space (electrodes) and time (in milliseconds or samples). To capture spatial and temporal metrics, we use data-driven methods for ERP pattern analysis (Figure 1, Box [1]). and metric generation (Figure 1,Box [2]). The metrics provide a compact summary of ERP patterns and are expressed in RDF (resource description framework) using terms from the NEMO ontology. The metric and RDF generation processes are fully automated.

In addition to spatial and temporal features, which are automatically extracted using the NEMO ERP Toolkit, we capture experiment metadata through the NEMO portal (Figure 1, Box 3; see Section 4.2 for details). Once ERP spatial, temporal, and functional (experimental) features have been expressed in RDF, the NEMO ontology can be used to classify and label the spatiotemporal patterns that are represented by these features (see Refs [1-3] for further details). Thus, ontology-based labeling of data (via RDF) gives a powerful way to link ERP data to a larger base of information that can be used for classification and integration.

2 RDF2: Data Analysis (aka "Workflow") Provenance

1 RDF1: Data (aka "Metric Data")

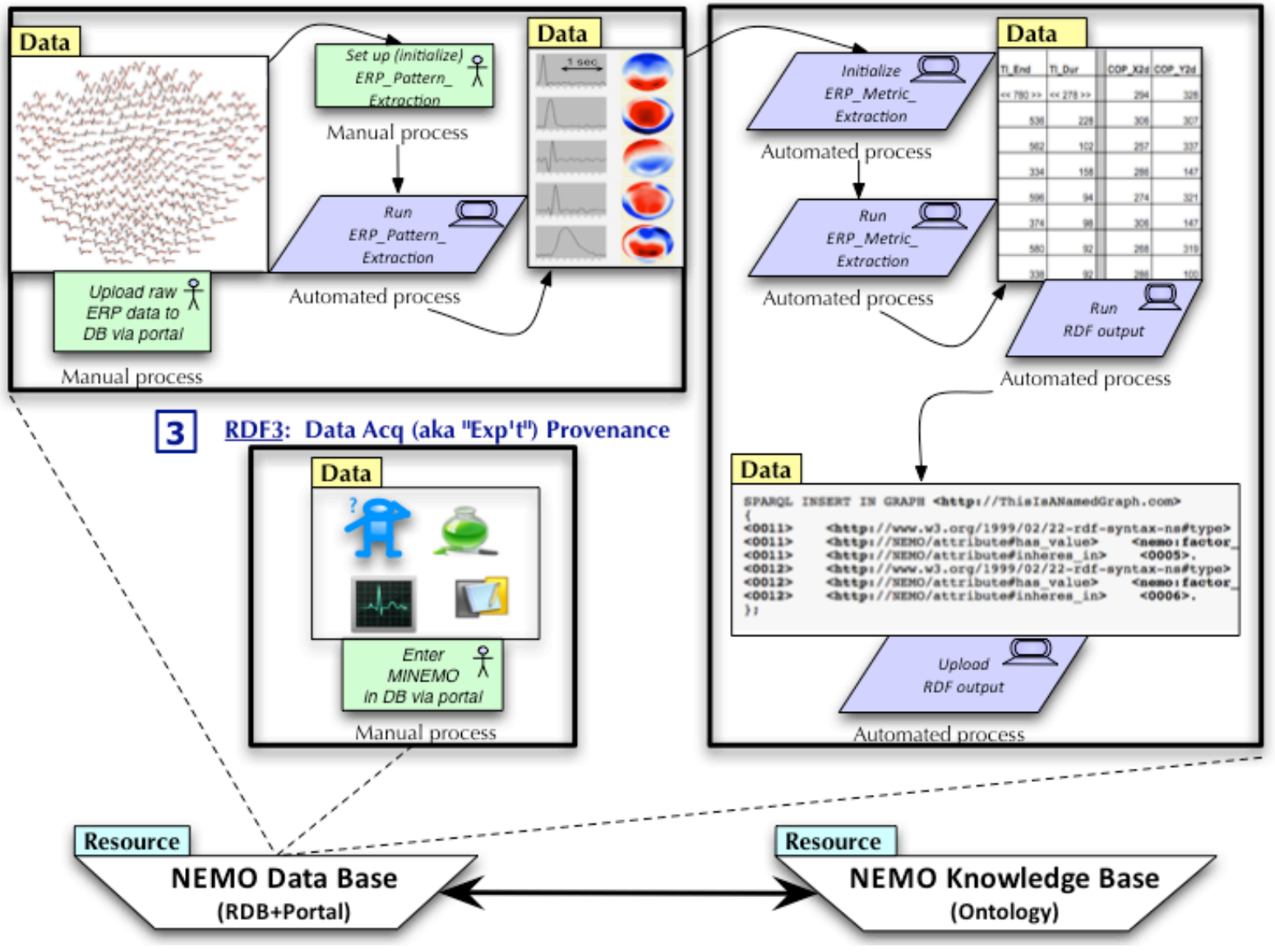

Figure 1. Box [1]: Data-driven ERP pattern analysis. Box [2]: Generation of spatial and temporal metrics (expressed in RDF). Box [3]: Use of NEMO portal for entry of experiment metadata. 


\section{The NEMO portal: Annotation of ERP experiment metadata}

The main motivation for MINEMO is to provide a controlled vocabulary for annotation of ERP metadata. In previous work, we showed that both temporal and spatial metrics are needed for accurate classification of ERP data $[19,20]$. In addition, however, many ERP patterns are also characterized by the functional (i.e., experimental) context in which the data were acquired. For example, the topographic distribution of the wellknown N100 pattern is different for visual and auditory stimuli, reflecting activation of distinct neural networks in visual and auditory processing [21]. Similarly, the visual evoked N100 is often greater over the left side of the scalp in response to words, but is bilateral or right-lateralized in response to faces [22].

Ideally, experiment metadata should be provided when a dataset is submitted for NEMO ERP analysis. To this end, we created a web application (the NEMO portal), database and services that enable NEMO users to record their experiment metadata online through a simple web interface at the same time that they upload their actual datasets to the NEMO database. The NEMO portal [23] is built around three objects: Users, Laboratories, and Experiments. Each user represents an individual researcher and is also a member of some laboratory. In order to access most functions within the portal, a researcher must obtain a user account. Once an account is created, the researcher can login to the portal and start the process of creating an experiment entry. When creating an experiment entry, the researcher enters MINEMO information through a series of HTML forms. The metadata fields correspond with entities in the NEMO ontology; in other words, we capture through the portal a complete description of an experiment, consistent with the standard established by the NEMO ontology and by the MINEMO checklist. To assist portal users, we created a tooltip mechanism that overlays ontology information directly on any form item when the user hovers their mouse pointer over that item. If the user is unsure of the meaning of an item while filling out a form, they can quickly lookup the ontology definition of that item using the tooltip overlay, as depicted in Figure 2.

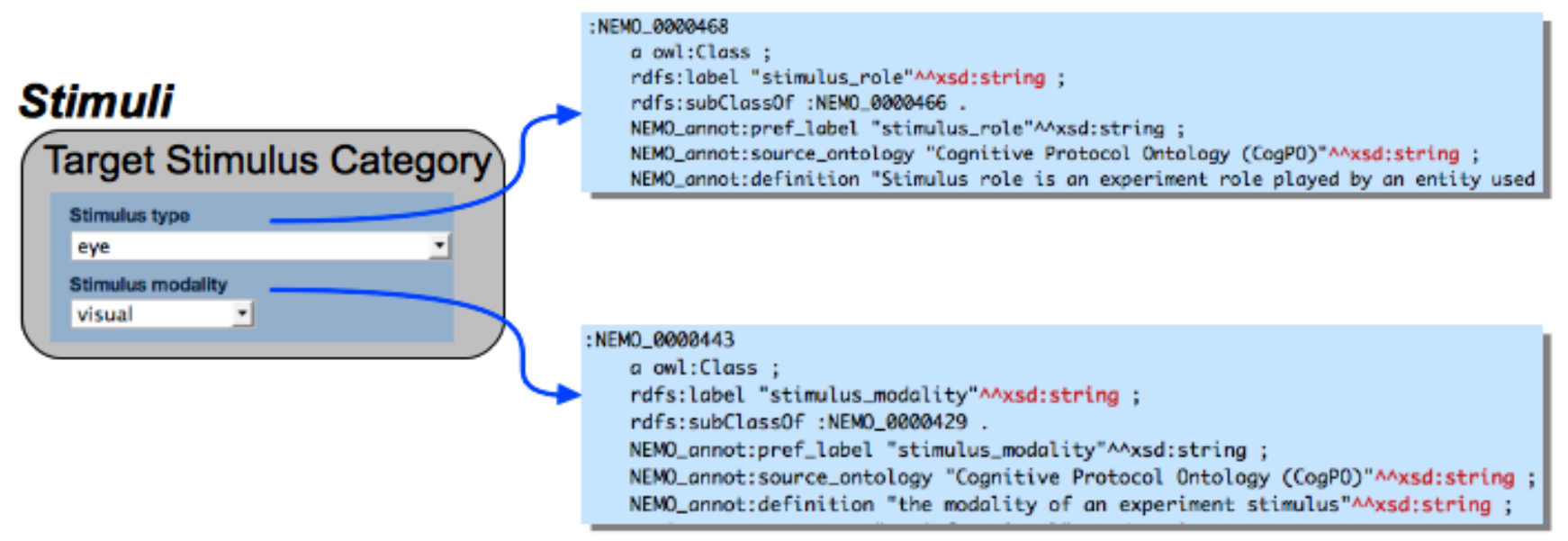

Figure 2. Sample metadata field in NEMO portal and illustration of "tooltips."

All form information is saved to an SQL database. Saved experiments can be edited at any time, and previously entered information can be copied and modified for inclusion in new entries, to reduce redundant data entry.

Figure 3 gives a conceptual overview of how the NEMO portal and database make contact with the NEMO ontology and MI checklist. Notice that experiment metadata are written out to $\mathrm{RDF}$
(Figure 3, bottom right) and are then combined with the RDF representation of spatial and temporal metrics, which are stored in a Results Database.

Once experiment metadata have been captured in $\mathrm{RDF}$, they can then be combined with the spatial and temporal metrics to provide a complete description of ERP patterns for input to classification and meta-analysis. 


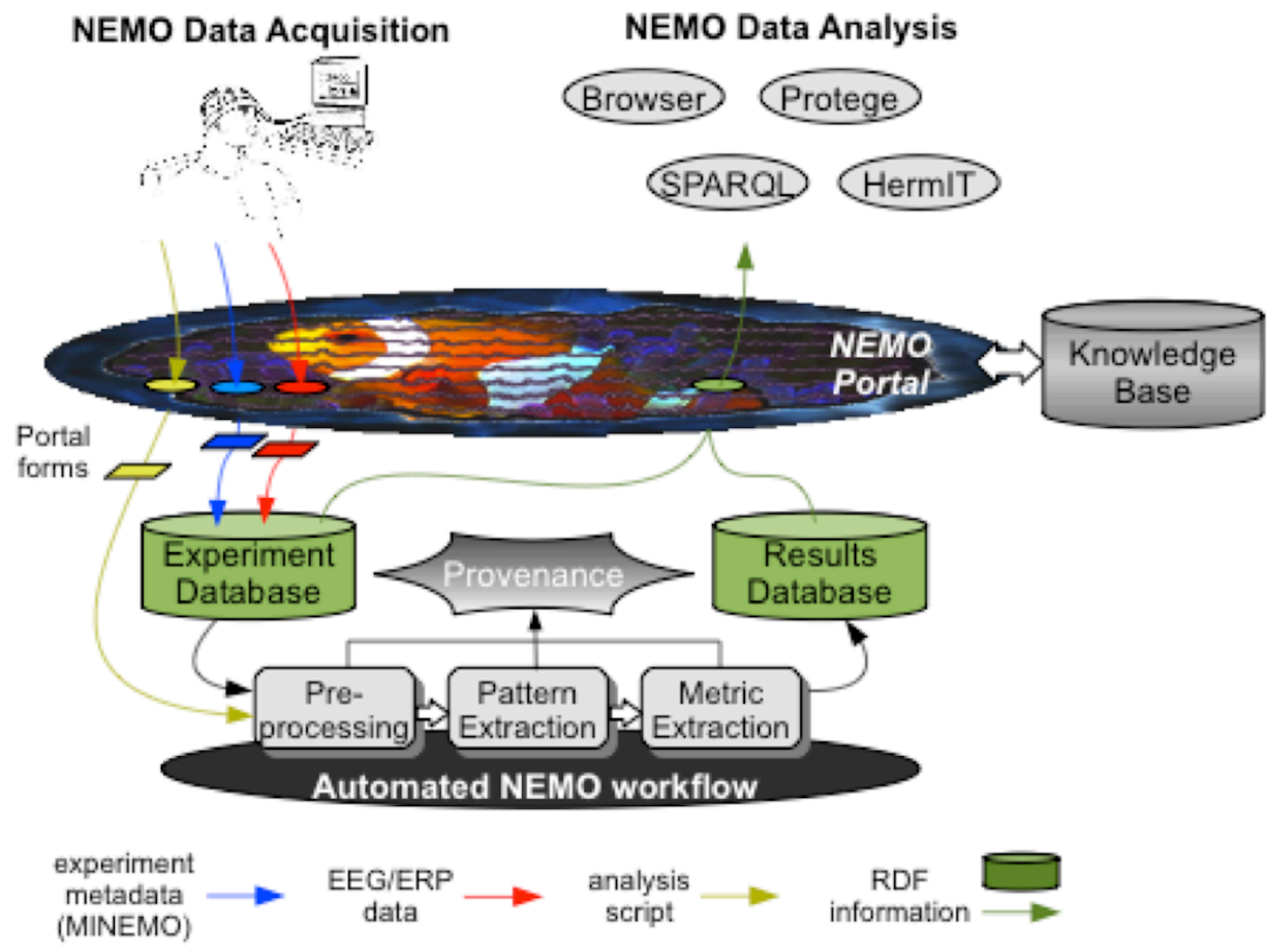

Figure 3. Overview of links between NEMO portal, database, ontology and MI checklist.

\section{Summary and conclusion}

\section{Community participation}

NEMO is a relatively new project, and our initial efforts have been focused on developing and testing ERP ontologies and ontology-based tools for analysis. Our next step will be to apply these methods and tools to high-dimensional ERP datasets (with 100 EEG sensors or more) that have been collected across our research sites and to report findings from our first cross-lab, crossexperiment meta-analysis.

Once we have provided this important "proof of concept," we will solicit feedback from the wider clinical and cognitive neuroscience communities. All NEMO ontology (owl) files and NEMO ERP analysis and RDF generation code are freely available from our source forge repository [24]. Documentation is available from our Wiki [25]. We encourage members of the community to browse and download these resources and to provide feedback to our development team. To this end, we have established a public listserv [26].

\section{Future work}

Future work will extend the NEMO portal to support data analysis workflows and to capture workflow provenance in the process. To support this effort, we will adopt parts of two provenance ontologies, the Open Provenance Model (OPM [27]; ) and Provenir ( [28]). The NEMO portal will then be used to store workflow provenance in database structures that are mapped to the NEMO ontology. We think that capturing the context for data acquisition and analysis, and the rich set of parameters that are associated with these processes, will be critically important for accurate comparison of ERP patterns that are the result of different analysis workflows. 


\section{Summary and Conclusion}

In conclusion, we have described the development and application of MINEMO (Minimal Information for Neural ElectroMagnetic Ontologies), a checklist for description of event-related potentials (ERP) studies. MINEMO extends MINI (Minimal Information for Neuroscience Investigations) to the ERP domain. Checklist terms are explicated in $N E M O$, a formal ontology that is designed to support ERP data sharing and integration. MINEMO is also linked to an ERP database and web application (the NEMO portal), which enables the capture of experimental provenance through a direct implementation of MINEMO [29]. Each item on the MINEMO list is encoded in an HTML form on the NEMO Portal and stored in a SQL database. The database also stores metadata entries in RDF (Resource Description Framework), along with summary metrics, i.e.,

\section{References}

1. Frishkoff GA, Frank R, Rong J, Dou D, Dien J, Halderman L. (2007). A framework to support automated ERP pattern classification and labeling. Computational Intelligence and Neuroscience, vol. 2007, Article ID 14567, 13 pages.

2. Frishkoff G, Frank R, LePendu P, Nikolic S. (2011). Ontology-based Analysis of Event-Related Potentials. Proceedings of thelnternational Conference on Biomedical Ontology (ICBO'11).

3. Frishkoff GA, Dou D, Frank R, LePendu P, Liu H. (2009). Development of Neural Electromagnetic Ontologies (NEMO): Representation and integration of event-related brain potentials. Proceedings of the International Conference on Biomedical Ontologies (ICBO09).July 24-26, 2009. Buffalo, NY.

4. Taylor CF, Field D, Sansone SA, Aerts J, Apweiler R, Ashburner M, Ball CA, Binz PA, Bogue M, Booth $\mathrm{T}$, et al. Promoting coherent minimum reporting guidelines for biological and biomedical investigations: the MIBBI project. Nat Biotechnol 2008; 26:889-896. PubMed doi:10.1038/nbt.1411

5. Gibson F, Overton PG, Smulders TV, Schultz SR, Eglen SJ, Ingram CD, Panzeri S, Bream P, Sernagor E, Cunningham M et al. (2010). Minimum Information about a Neuroscience Investigation (MINI): Electrophysiology. Nature Precedings. spatial and temporal metadata. Together these spatial, temporal, and functional metadata provide a complete description of ERP data and the context in which these data were acquired. The RDF files then serve as inputs to ontology-based labeling and meta-analysis.

We believe this approach can lead to important new discoveries, for example, by enabling us to compare neural patterns across study paradigms that have distinct but overlapping experimental contexts (e.g., studies of episodic and semantic memory and word comprehension [1]). Given the active investment in similar activities across the sciences, there is a strong possibility that these efforts could lead to knowledge integration, or consilience, across traditional boundaries. The path to this outcome will require dedicated work and collaboration of many groups; the payoff, though, seems well worth the effort.

6. Poldrack RA, Fletcher PC, Henson RN, Worsley KJ, Brett M, Nichols TE. Guidelines for reporting an fMRI study. Neuroimage 2008; 40:409-414. $\underline{\text { PubMed doi:10.1016/j.neuroimage.2007.11.048 }}$

7. Duncan CC, Barry RJ, Connolly JF, Fischer C, Michie PT, Näätänen R, Polich J, Reinvang I, Van Petten C. Event-related potentials in clinical research: guidelines for eliciting, recording, and quantifying mismatch negativity, P300, and N400. Clin Neurophysiol 2009; 120:1883-1908. PubMed doi:10.1016/j.clinph.2009.07.045

8. Eimer M. Methodological issues in event-related brain potential research. Behav Res Methods Instrum Comput 1998; 30:3-7. doi:10.3758/BF03209411

9. Picton TW, Bentin S, Berg P, Donchin E, Hillyard SA, Johnson R, Jr., Miller GA, Ritter W, Ruchkin DS, Rugg MD, Taylor MJ. Guidelines for using human event-related potentials to study cognition: recording standards and publication criteria. Psychophysiology 2000; 37:127-152. PubMed doi:10.1111/1469-8986.3720127

10. Laird AR, Lancaster JL, Fox PT. BrainMap: the social evolution of a human brain mapping database. Neuroinformatics 2005; 3:65-78. PubMed doi:10.1385/NI:3:1:065

11. Hill, D. P., Smith, B., McAndrews-Hill, M. S., \& Blake, J. A. (2008). Gene Ontology annotations: What they mean and where they come from. BMC Bioinformatics, 9 Suppl 5. 
12. Fox PT, Laird AR, Fox SP, Fox PM, Uecker AM, Crank M, Koenig SF, Lancaster JL. BrainMap taxonomy of experimental design: description and evaluation. Hum Brain Mapp 2005; 25:185-198. PubMed doi:10.1002/hbm.20141

13. Laird AR, Eickhoff SB, Kurth F, Fox PM, Uecker AM, Turner JA, Robinson JL, Lancaster JL, Fox PT. ALE Meta-Analysis Workflows Via the Brainmap Database: Progress Towards A Probabilistic Functional Brain Atlas. Front Neuroinformatics 2009; 3:23. PubMed

14. Larson SD, Martone ME. Ontologies for Neuroscience: What are they and What are they Good for? Frontiers in Neuroscience 2009; 3:6067. PubMed doi:10.3389/neuro.01.007.2009

15. Bug WJ, Ascoli GA, Grethe JS, Gupta A, Fennema-Notestine C, Laird AR, Larson SD, Rubin D, Shepherd GM, Turner JA, et al. The NIFSTD and BIRNLex vocabularies: building comprehensive ontologies for neuroscience. Neuroinformatics 2008; 6:175-194. PubMed doi:10.1007/s12021-008-9032-Z

16. Gardner D, Akil H, Ascoli GA, Bowden DM, Bug W, Donohue DE, Goldberg DH, Grafstein B, Grethe JS, Gupta A, et al. The neuroscience information framework: a data and knowledge environment for neuroscience. Neuroinformatics 2008; 6:149-160. PubMed doi:10.1007/s12021$\underline{008-9024-Z}$

17. Gupta A, Bug W, Marenco L, Qian X, Condit C, Rangarajan A, Müller HM, Miller PL, Sanders B, Grethe JS, et al. Federated access to heterogeneous information resources in the Neuroscience Information Framework (NIF). Neuroinformatics 2008; 6:205-217. PubMed doi:10.1007/s12021-008-9033-y

18. Turner JA, Laird AR. The Cognitive Paradigm Ontology: Design and Application. Neuroinformatics 2011; (In press). PubMed doi:10.1007/s12021-011-9126-x

19. Liu H, Frishkoff GA, Frank R, Dou D. (2010).Ontology-based mining of brainwaves: sequence similarity technique formapping alternative descriptions of patterns in eventrelated potentials(ERP) data. Proceedings of the 14th Pacific-Asia Conference on KnowledgeDiscovery and Data Mining (PAKDD'10), 12 pages.

20. Liu, H., Frishkoff, G., Frank, R. M. F., \& Dou, D. (2011, in revision). Integration of Human Brain Data: Metric and Pattern Matching across
Heterogeneous ERP Datasets. Journal of Neurocomputing.

21. Alho K, Woods DL, Algazi A, Naatanen R. Intermodal selective attention. II. Effects of attentional load on processing of auditory and visual stimuli in central space. Electroencephalogr Clin Neurophysiol 1992; 82:356-368. PubMed doi:10.1016/0013-4694(92)90005-3

22. Maurer U, Rossion B, McCandliss BD. Category specificity in early perception: face and word $\mathrm{n} 170$ responses differ in both lateralization and habituation properties. Front Hum Neurosci 2008; 2:18. PubMed doi:10.3389/neuro.09.018.2008

23. The NEMO portal. http://portal.nemo.nic.uoregon.edu

24. Repositories SCM. http://nemoontologies.svn.sourceforge.net/viewvc /nemoontologies

25. Wiki NEMO. http://nemo.nic.uoregon.edu/wiki/NEMO

26. NEMO public listserv. nemoontologiespublic@lists.sourceforge.net

27. Moreau L, Clifford B, Freire J, Futrelle J, Gil Y, Groth P, Futrelle J, Kwasnikowska N, Miles S, Missie P et al. (2010). The open provenance model core specification (v1.1). Future Generation Computer Systems.

28. Sahoo SS, Sheth A. (2009, Oct 15-17, 2009). Provenir ontology: Towards a Framework for eScience Provenance Management. Paper presented at the Microsoft eScience Workshop, Pittsburgh, PA.

29. Minimal Information for Neural ElectroMagnetic Ontologies. http://mibbi.org/index.php/Projects/MINEMO 


\section{Appendix A: MINEMO Checklist}

1. Research lab (General features)
a. * Lab ID
b. *Institution
c. * Principal investigator (PI)
d. PI email address
e. PI mailing address

2. Experiment (general features)
a. *Experiment ID
b. *Experimental paradigm(s)
c. Start date for data collection
d. End date for data collection

3. Publication
a. *Publication ID
b. *Publication type
c. First author
d. Publication date
e. Title of paper
f. Book or Journal
g. *DOI or File Location (Path)

4. Study subjects (group

characteristics)
a. *Subject group ID
b. *Diagnostic classification
c. *Genus
d. *Species
e. *Age (average)
f. *Gender (\#male, female subjects)
g. *Handedness (\#RH, LH subjects)
h. ${ }^{*}$ Native language (modal)

5. Experiment condition
a. ${ }^{*}$ Condition ID
b. * Experiment condition
c. *Experiment task (Instructions)
d. Number of trials per condition

6. Stimulus Presentation
a. *Stimulus type ID
b. Stimulus presentation device
c. Stimulus presentation software
d. *Target stimulus type
e. *Target stimulus modality
f. Target stimulus duration
g. Prime stimulus type (if relevant)
h. Prime stimulus modality (if relevant)
i. Prime stimulus duration (if relevant)
j. Prime-Target ISI (if relevant)
k. Prime-Target SOA (if relevant)

7. Behavioral data collection
a. *Response type ID
b. Response collection device
c. Response presentation software
d. *Response type
e. *Response modality
f. Response deadline
g. Response accuracy (average)
h. Response time (average)

8. EEG Data Collection

a. Electrode array (Manufacturer)

b. *Electrode array (Layout)

c. Reference electrode

d. Ground (noise) electrode

e. Scalp-to-Electrode impedance threshold

f. Amplifier gain

g. Amplifier input impedance

h. *Sampling rate

i. Amplifier filter setting(s)

9. EEG/ERP Data preprocessing

a. Digital filter transformation(s)

b. Digital cleaning method(s)

c. *ERP event

d. *ERP epochlength (in $\mathrm{ms}$ )

e. ${ }^{*}$ ERP baseline (pre-Target) duration

f. * Offline reference

10. EEG/ERP Data file

a. Data file contents (EEG data type)

b. Data file format

c. Data file location (URI)

* Denotes required field for entry of data in NEMO portal 


\section{Appendix B: MINEMO Term Definitions and Ontology URI}

Table 1. Research Lab

\begin{tabular}{lll}
\hline Term & URI (NEMO) & Definition \\
\hline Lab ID & NEMO_7431000 & $\begin{array}{l}\text { A unique identifier for the facility where data were collected } \\
\text { Institution }\end{array}$ \\
NEMO_1725000 & $\begin{array}{l}\text { University or other institution (hospital, company) where data were } \\
\text { collected }\end{array}$ \\
Principal Investigator (PI) & OBI_0000103 & Person responsible for the overall conduct of the study \\
PI email address & NEMO_8251000 & Email address for PI \\
PI postal address & NEMO_0670000 & Postal address for PI \\
\hline
\end{tabular}

Table 2. Experiment (General Features)

\begin{tabular}{lll}
\hline Term & URI (NEMO) & Definition \\
\hline Experiment ID & NEMO_0000537 & A unique identifier for the experiment \\
Start date & NEMO_8539000 & Start date for experiment (YYYY) \\
End date & NEMO_0917000 & End date for experiment (YYYY) \\
\hline
\end{tabular}

Table 3. Documentation

\begin{tabular}{lll}
\hline Term & URI (NEMO) & Definition \\
\hline $\begin{array}{l}\text { Publication type } \\
\text { First author }\end{array}$ & BRO: Narrative_Resource & $\begin{array}{l}\text { Type of document (journal article, book chapter, } \\
\text { unpublished manucript, etc.) }\end{array}$ \\
Publication date & NEMO_1264000302 & $\begin{array}{l}\text { First author (family name) on document } \\
\text { Title of paper }\end{array}$ \\
Title of volume & NEMO_1010000 & $\begin{array}{l}\text { Date of publication (YYYY) } \\
\text { Title of paper }\end{array}$ \\
DOI or File location & NEMO_2062000 & $\begin{array}{l}\text { Title of volume (e.g., journal, } \\
\text { book, or conference proceeding) } \\
\text { Label that denotes the } \\
\text { unique location of the document }\end{array}$ \\
\hline
\end{tabular}

Table 4. Study Subjects (Group Characteristics)

\begin{tabular}{lll}
\hline Term & URI (NEMO) & Definition \\
\hline Subject Group ID & NEMO_2014000 & Subject group identifier \\
Diagnostic Classification & NEMO_5159000 & Default classification is "normal" \\
Genus & NEMO_5621000 & Default category is "homo" \\
Species & NEMO_3454000 & Default category is "sapiens" \\
Age (average) & NEMO_2506000 & Average age (in years) of study group \\
Gender (count) & NeMO_6503000 & Number of male, female subjects \\
Handedness (count) & NEMO_7467000 & Native language(s) spoken by subjects \\
\hline
\end{tabular}


Table 5. Experiment Context, Paradigm

\begin{tabular}{lll}
\hline Term & URI (NEMO) & Definition \\
\hline Experiment Paradigm & NEMO_0000379 & $\begin{array}{l}\text { A factorial design (specification of study variables) that is } \\
\text { implemented in an experiment (cf. BrainMapLex) }\end{array}$ \\
ECI Software & NEMO_1752000 & $\begin{array}{l}\text { Software application that controls timing of stimulus presentation } \\
\text { and recording of behavioral and neural responses }\end{array}$ \\
Experiment Condition & NEMO_0000382 & $\begin{array}{l}\text { A recognizable set of experiment features (stimulus and response } \\
\text { type, instructions) }\end{array}$ \\
Task (Instructions) & NEMO_0000383 & $\begin{array}{l}\text { Explicit direction that guides subject behavior during experiment } \\
\text { (cf. BrainMapLex) }\end{array}$ \\
Number trials (per condition) & NEMO_6697000 & The number of trials in an experiment condition \\
\hline
\end{tabular}

Table 6. Stimulus Presentation

\begin{tabular}{|c|c|c|}
\hline Term & URI (NEMO) & Definition \\
\hline Stimulus presentation device & NEMO_8446000 & A device that is used forstimulus presentation \\
\hline Target stimulus (type) & NEMO_5065000 & $\begin{array}{l}\text { Role of stimulus that has features which study participants } \\
\text { areasked to attend to and/or select for further processing }\end{array}$ \\
\hline Target stimulus modality & NEMO_0000443 & Modality of target stimulus (visual, auditory, etc.) \\
\hline Target stimulus duration & NEMO_3331000 & Duration of target stimulus (in ms.) \\
\hline Prime stimulus (type) & NEMO_2367000 & $\begin{array}{l}\text { Stimulus that precedes the target and is intended to } \\
\text { affecttarget processing }\end{array}$ \\
\hline Prime stimulus modality & NEMO_0000443 & Modality of prime stimulus (visual, auditory, etc.) \\
\hline Prime stimulus duration & NEMO_5109000 & Duration of prime stimulus (in ms.) \\
\hline Prime-Target ISI & NEMO_8410000 & $\begin{array}{l}\text { Time interval that separates offset of prime and onset of target } \\
\text { stimulus }\end{array}$ \\
\hline
\end{tabular}

Table 7. Behavioral Data Collection

\begin{tabular}{|c|c|c|}
\hline Term & URI (NEMO) & Definition \\
\hline Response collection device & NEMO_0000503 & A device that is used to record behavioral data \\
\hline Response type & NEMO_0000467 & $\begin{array}{l}\text { A behavioral process that occurs an experimentin } \\
\text { response to stimulus }\end{array}$ \\
\hline Response modality & NEMO_0000756 & $\begin{array}{l}\text { Class of body parts used to perform actions that can play } \\
\text { the role of a response (cogPO) }\end{array}$ \\
\hline Response deadline & NEMO_2473000 & $\begin{array}{l}\text { The maximum time that is allowed for an experiment } \\
\text { response }\end{array}$ \\
\hline Accuracy (average) & NEMO_0000431 & The accuracy (correctness) of an experimental response \\
\hline Response time (average) & NEMO_0000433 & The latency (onset time) of an experimental response \\
\hline
\end{tabular}




\begin{tabular}{|c|c|c|}
\hline Term & URI (NEMO) & Definition \\
\hline Electrode array (manufacturer) & NEMO_3240000 & $\begin{array}{l}\text { Name of company that produced EEG sensors and } \\
\text { sensor array }\end{array}$ \\
\hline Electrode array (layout) & NEMO_6227000 & $\begin{array}{l}\text { Number of EEG sensors and topographic layout of } \\
\text { sensors }\end{array}$ \\
\hline Recording reference & NEMO_6771000 & $\begin{array}{l}\text { 10-20 location of electrode(s) used as EEG } \\
\text { recording reference }\end{array}$ \\
\hline Ground (noise) electrode & NEMO_4335000 & $\begin{array}{l}\text { 10-20 location of electrode(s) used as isolated } \\
\text { common (aka "ground") }\end{array}$ \\
\hline Scalp-to-electrode impedance threshold & NEMO_6541000 & Maximum scalp-to-electrode impedance (in $\mathrm{Hz}$ ) \\
\hline Amplifier gain & & Resolution (in bits/microvolt) \\
\hline Amplifier input impedance & NEMO_2655000 & Input impedance to amp (in $\mathrm{Hz}$ ) \\
\hline Temporal sampling rate & NEMO_2585000 & Sampling rate (in ms) \\
\hline Amplifier filter setting & NEMO_1142000 & Analog bandpass (in $\mathrm{Hz}$ ) \\
\hline
\end{tabular}

Table 9. EEG/ERP Data File

\begin{tabular}{lll}
\hline Term & URI (NEMO) & Definition \\
\hline Data file contents & NEMO_2662000 & Type of data (continuous, segmented, or averaged EEG) \\
Data file format & NEMO_1194000 & Data file format (.raw, .txt, etc.) \\
Data file location & NEMO_3087000 & Unique resource identifier or file path \\
\hline
\end{tabular}

Table 10. EEG/ERP Data Preprocessing

\begin{tabular}{lll}
\hline Term & URI (NEMO) & Definition \\
\hline Digital filter transformation & NEMO_7669000 & $\begin{array}{l}\text { Offline removal of signal above or below a certain } \\
\text { frequency level } \\
\text { Data cleaning transformation }\end{array}$ \\
NEMO_4273000 & $\begin{array}{l}\text { Offline removal of any signal that is not of interest to the } \\
\text { researcher } \\
\text { The role ofan event (e.g., stimulus onset)that is used } \\
\text { forEEG averaging }\end{array}$ \\
ERP epoch length & NEMO_3620000 & $\begin{array}{l}\text { The durationof an ERP, where the time of the event is } \\
\text { designated as time zero. }\end{array}$ \\
ERP baseline length & NEMO_6232000 & $\begin{array}{l}\text { The durationof ERP baseline (by default, the end of the } \\
\text { baseline is the onset of the ERP) }\end{array}$ \\
Offline reference & NEMO_0000321 & Offline schema used for re-reference
\end{tabular}

${ }^{1}$ An alternative to the use of a controlled vocabulary is to discover mappings between data annotations $[19,20]$. However, this process is nontrivial, and its success depends on the nature and amount of variability in the data. 